\title{
A História da Computação através de um Museu Virtual Interativo utilizando Mundos Virtuais 3D
}

\author{
Andréia Solange Bos ${ }^{1}$, Pablo Dalssasso ${ }^{1}$, Luis Henrique C. Rosa $^{1}$, Giliane Bernardi ${ }^{1}$, Felipe \\ Martins Müller ${ }^{1}$, Andre Zanki Cordenonsi ${ }^{2}$ \\ ${ }^{1}$ Departamento de Computação Aplicada - Universidade Federal de Santa Maria (UFSM) \\ Av. Roraima 1000 - Santa Maria - RS - Brasil \\ ${ }^{2}$ Departamento de Documentação- Universidade Federal de Santa Maria (UFSM) \\ Av. Roraima 1000 - Santa Maria - RS - Brasil \\ andreia.bos@gmail.com, \{dalsasso, lhcr, giliane, andrezc $\} @$ inf.ufsm.br
}

\begin{abstract}
D Virtual Worlds have been increasingly used in the educational context, aiming to increase the interaction and engagement of students with academic activities. In this context, this paper aims to present a $3 D$ virtual museum that presents the history of computing, developed by using OpenSimulator virtual environment. By proposing the use of $3 D$ virtual worlds in teaching and learning, is intended to achieve greater interaction between students and especially between students and the study object, in this case the artifacts of the history of computing, in order to contribute to the construction of new educational approaches, and especially to the motivation of students for technological area.
\end{abstract}

Resumo. Mundos Virtuais $3 D$ têm sido cada vez mais utilizados no contexto educacional, buscando aumentar a interação e engajamento dos estudantes junto às atividades acadêmicas. Neste contexto, este artigo tem como objetivo apresentar um museu virtual $3 D$ que apresenta a história da computação, desenvolvido através do ambiente virtual OpenSimulator. Ao propor a utilização de mundos virtuais $3 D$ no processo de ensino e aprendizagem, pretende-se alcançar uma maior interação entre os estudantes e, em especial dos estudantes com o objeto de estudo, neste caso os artefatos da história da computação, buscando contribuir para a construção de novas abordagens educacionais e, principalmente, na motivação dos estudantes pela área tecnológica.

\section{Introdução}

Usualmente, tanto cursos de computação quanto áreas afins possuem em seus currículos uma disciplina de introdução à área da computação, que busca explorar a história da computação e sua relação com as principais formas de utilização e aplicação de computadores, apresentando aos estudantes uma visão geral e abrangente do escopo que envolve a computação nos mais diversos segmentos da sociedade. Os principais livros na área de introdução à computação também abordam estes temas, bem como aspectos do funcionamento de um computador e linguagens de programação (Forouzan, 2012; Fedeli, 2003).

Uma das primeiras aulas trabalhadas junto aos estudantes trata dos aspectos da história da computação. Segundo Berry et al. (2011), o ensino da história da computação é amplamente aceito no que tange a ajudar os estudantes da área a entenderem e conhecerem seu futuro campo 
de atuação. Ainda, alguns autores destacam que é fundamental ao estudante de computação possuir uma compreensão dos aspectos técnicos e culturais de sua área, de seu passado e presente, como fonte importante para seus estudos acadêmicos no decorrer do curso (Medina, 2004) (Giangrandi, P. and Mirolo, C, 2006).

Porém, do ponto de vista educacional, o que se observa, na maioria dos casos, são apresentações expositivas, por meio de slides, que traçam a trajetória da computação nas diferentes décadas de sua evolução. Neste contexto, diferentes abordagens têm sido propostas para agregar às aulas consideradas "tradicionais", entre elas o uso de simulações, jogos computacionais e ambientes virtuais (Chengwei et al. 2011; Valente e Mattar, 2007).

Considerando o foco deste trabalho, que trata da abordagem da história da computação em cursos de graduação, destaca-se como uma possibilidade apresentar de forma mais interativa esta evolução e artefatos de cada década de avanços tecnológicos. Uma das possibilidades é a utilização de mundos virtuais 3D onde os estudantes possam visualizar artefatos e interagir com os mesmos. Neste contexto, surge a proposta de criar um museu virtual imerso em um Mundo Virtual 3D, onde os estudantes possam realizar uma visitação virtual pela história da computação interagindo com os diferentes artefatos de cada época. Este artigo apresenta o projeto e desenvolvimento do mesmo.

\section{Museus Virtuais}

Museu é um nome genérico que se dá a um conjunto de manifestações simbólicas da sociedade humana, em diferentes tempos e espaços. Uma destas formas de exposição são os museus virtuais, que compreendem uma coleção de artefatos eletrônicos e apresentam como uma das vantagens em relação aos museus tradicionais a reprodução digital de objetos reais, que ainda existem ou não, oferecendo a possibilidade de observar e interagir com as obras e objetos, além de disponibilizar diversos recursos multimídia (Chengwei et al., 2011).

Museus virtuais podem ser usados no processo de ensino-aprendizagem, proporcionando um novo espaço para socialização e permitindo aos usuários visitarem, interagirem e manipularem seus artefatos de forma mais flexível. Segundo Lévy (2000), museus virtuais trazem possibilidades de serem usados em ambientes educacionais desempenhando um papel fundamental no aumento da motivação e na melhoria do processo de aprendizagem.

Considerando estas possibilidades, é possível conceber a utilização de um ambiente como este para a exploração de conceitos e história da computação tanto em aulas presenciais como a distância. Possibilitar aos estudantes percorrerem corredores de um museu, interagindo com artefatos e objetos históricos de diferentes décadas da evolução da computação pode tornar as aulas mais dinâmicas e motivadoras.

\section{Mundos Virtuais 3D e seu potencial na Educação}

Mundos virtuais 3D são ambientes online, onde as pessoas podem interagir de maneira comparável ao mundo real, seja para o trabalho ou lazer, tendo em vista que a partir de uma personificação do indivíduo (avatar) no mundo virtual, o usuário pode se comunicar via canal de voz, mensagens de texto e exibir expressões corporais (Bainbridge, 2010). Assim, instituições acadêmicas e empresas têm explorado os benefícios de ambientes imersivos onde os participantes podem interagir uns com os outros, bem como, com o professor, mesmo que estejam fisicamente a milhares de quilômetros de distância (Wankel and Kingsley, 2009). 
No segmento de Mundos Virtuais 3D as possibilidades de imersão em ambientes como Second Life (SL) e OpenSimulator têm sido utilizadas para o desenvolvimento de ambientes colaborativos de realidade virtual para uso educacional. Segundo Azevedo e Elia (2011), essas ferramentas computacionais proporcionam aos usuários novas formas de aprendizagem permitindo a construção de cenários e recursos que recriam/simulam com mais fidedignidade o mundo real. Ainda, para (Osório et al. 2004), o paradigma 3D oferece a possibilidade de representar a informação de um modo realístico, organizando-a de uma maneira espacial e tornando sua visualização mais intuitiva por ser mais natural ao ser humano.

Por fim, (Schlemmer et al., 2008) afirmam que a aprendizagem acontece na interação do sujeito com o objeto de conhecimento e com outros sujeitos, fazendo com que a interação seja um elemento essencial no processo educacional. Ao afirmarem isto, destacam que Mundos Virtuais 3D podem contribuir significativamente no processo de ensino e aprendizagem mediado por tecnologias, uma vez que estes podem trazer diferentes recursos como telepresença e avatares, diferente da abordagem convencional, que ocorre, quase que basicamente, por meio da linguagem textual.

\section{Trabalhos Correlatos}

É possível identificar diversas iniciativas de museus virtuais dedicados a apresentar a história e evolução da computação. O Museu Virtual da Informática (MVI), de Portugal (Almeida, 2006), apresenta a história da informática com base em páginas web. Dentro do museu, o estudante pode visualizar seu conteúdo através de diferentes formatos, tais como textos, imagens, sons, animações e vídeos. Outro museu que pode ser citado é o Museu do Computador, da Universidade Estadual de Maringá (http://www.din.uem.br/museu), desenvolvido pelo Departamento de Informática da universidade. O funcionamento do museu também é baseado em páginas web, que apresentam imagens e descrições de personalidades, descobertas e de invenções ao longo da história da computação.

Talvez o mais expressivo exemplo de museu na área de computação seja o Computer History Museum (http://www.computerhistory.org/), um museu físico que tem como principal objetivo apresentar artefatos e a história da era da informação, contendo mais de 1000 objetos, incluindo desde computadores da década de 40 e 50 até a história da internet e da computação móvel. No site do museu, é possível visitar o museu virtualmente, podendo visualizar diversos artefatos presentes fisicamente no museu, bem como obter informações sobre o mesmo.

O que se observa nos museus analisados é sua estrutura através de páginas web, com apresentação estática dos objetos, usualmente representados apenas por imagens em duas dimensões e textos explicativos acompanhando os mesmos. Poucas iniciativas possuem integração com algum tipo diferente de mídia, como vídeo, animações, simulações, entre outros. Ainda, não possuem qualquer tipo de integração com ambientes virtuais de ensino e aprendizagem, não são construídos em ambientes 3D e não privilegiam a interação entre seus visitantes ou com os artefatos do museu. Considerando estas colocações, a próxima seção apresenta o museu virtual proposto por este trabalho, que tem como objetivo focar nas potencialidades dos museus analisados, agregando possibilidades educacionais, tais como maior interação entre os estudantes, integração com ambiente virtual de ensino e aprendizagem, interação com os objetos maximizando a experiência virtual e diversidade de exibição dos conteúdos associados aos artefatos, por meio de diferentes mídias. 


\section{A História da Computação através de um Museu Virtual 3D}

O Museu da Computação para o ensino de introdução à computação traz a proposta de inserir o estudante em um museu virtual para auxiliar no ensino-aprendizagem nas disciplinas de introdução à computação dos cursos da área. Para o desenvolvimento do museu, foi escolhido o ambiente virtual OpenSimulator, que disponibiliza as ferramentas necessárias para criação, modelagem e animação dos objetos expostos. Para a escolha dos conteúdos e objetos a serem inseridos no museu, foi feito um levantamento de requisitos, através de pesquisas em currículos de universidades, sites de museus virtuais e livros sobre a história da computação. Após, foi realizado o projeto arquitetural do museu, para organizar fisicamente a disposição das galerias e salas do mesmo, assim como a disposição de seus artefatos, conteúdos e atividades propostas.

Ainda, foi definida a integração do museu virtual com ambiente virtual de ensino e aprendizagem Moodle ${ }^{1}$, através do módulo Sloodle ${ }^{2}$, de forma a permitir que o professor, ao final da visitação possa trazer atividades complementares aos estudantes, integrando o museu ao ambiente educacional. Esta integração permite a utilização de objetos, dentro do OpenSimulator, para exibição de conteúdos existentes no Moodle.

O Museu está dividido em um hall de entrada, quatro galerias de exposição e duas salas para convivência e estudos. No hall de entrada encontra-se um mapa que apresenta a estrutura do museu, com suas galerias e salas. A partir deste mapa, o usuário poderá se teletransportar diretamente para a sala desejada ou apenas obter uma orientação para sua visitação. Ainda, o hall disponibiliza um ambiente para o usuário assistir um vídeo de apresentação do museu, mostrando sua divisão e suas atrações. Na primeira galeria, a galeria da pré-história da computação (figura 01), encontram-se vários objetos de diferentes tempos, que representam as primeiras invenções humanas para contagem, exibição e manipulação de números, palavras, sons e imagens. Os objetos da galeria são interativos, apresentando animações, movimentos e imagens ao toque do usuário, que ajudam a entender seu funcionamento. Os mesmos estão organizados cronologicamente, começando com invenções e objetos criados na era anterior a Cristo, tais como a escrita cuneiforme, o relógio de sol, e o ábaco. Da era depois de Cristo, estão representados a régua de cálculo, a máquina de pascal, o tear programável, a máquina de diferenças, a máquina de escrever, o primeiro telefone, o telegrafone, o diodo e a televisão.

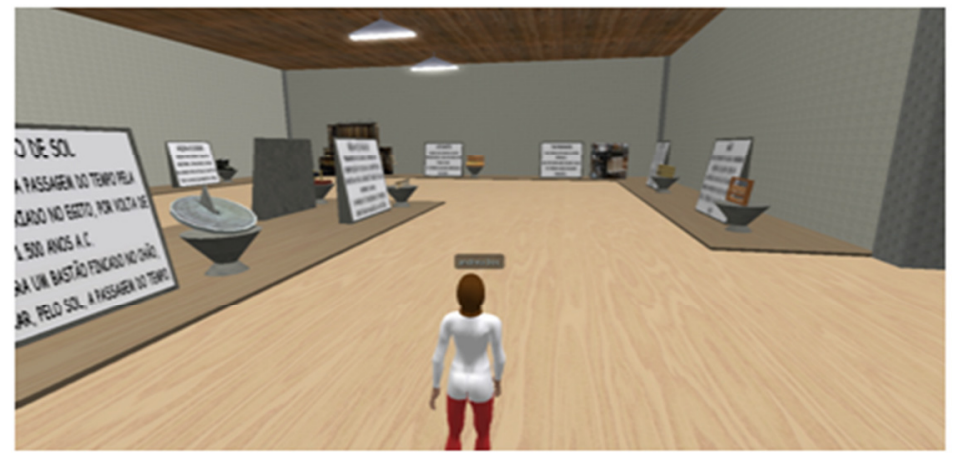

Figura 01. Galeria da pré-história da computação.

\footnotetext{
${ }^{1}$ Moodle - Plataforma de aprendizagem a distância baseada em software livre.

${ }^{2}$ Sloodle - Módulo de código aberto de integração da plataforma Moodle com o ambiente OpenSimulator ou Second Life, para gerenciamento de aprendizagem.
} 
As partes interativas dos artefatos da galeria são ativadas ao toque do visitante, possibilitando um melhor entendimento de seu funcionamento. Por exemplo, o ábaco (figura 02), ao ser tocado, abre um painel superior com uma animação que demonstra o modo como o objeto era utilizado; já a máquina de escrever (figura 03), realiza movimentos e reproduz sons, que simulam seu funcionamento.

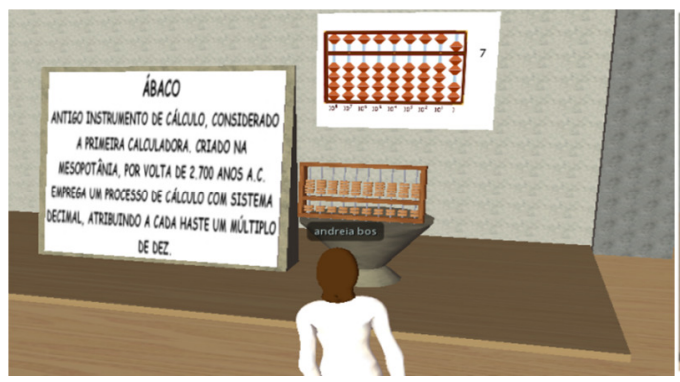

Figura 02. Animação do ábaco

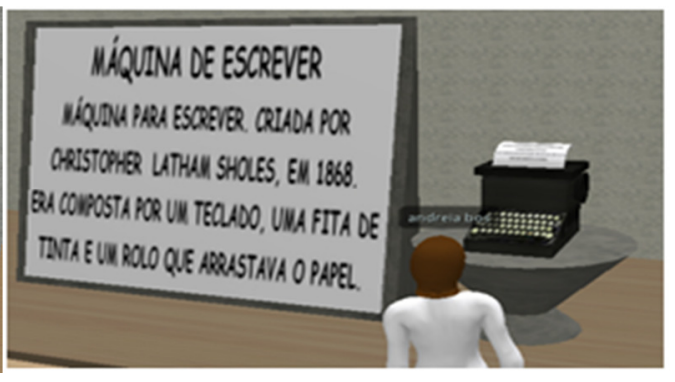

Figura 03. Animação da máquina de escrever

As demais galerias e salas do museu encontram-se em estágio de desenvolvimento. A galeria com as gerações dos computadores apresentará objetos que fazem parte das cinco gerações dos computadores (Forouzan, 2012); na terceira galeria, das personalidades da computação, serão expostos quadros com imagens de tais personalidades e informações sobre suas contribuições para a evolução da computação. A quarta galeria abordará a história das linguagens de programação, permitindo ao estudante conhecer e aprender algumas linguagens e suas evoluções, além de possibilitar a interação do visitante com alguns códigos simples.

A sala para estudos terá como objetivo a disponibilização de materiais adicionais, além dos apresentados durante a visitação, para o estudante obter maiores conhecimentos, bem como um espaço para avaliação dos conhecimentos adquiridos pelos estudantes, através da aplicação de atividades dentro do ambiente. Esta sala será integrada à plataforma Moodle, através do módulo Sloodle, já destacados nesta seção. A sala destinada ao ambiente de convivência e interação entre os alunos dentro do ambiente tem como propósito permitir aos estudantes discutirem os assuntos abordados durante a visita, em um momento maior de interação entre eles dentro do ambiente virtual, conforme o fariam se estivessem em uma sala de aula.

\section{Considerações Finais}

Este artigo teve como objetivo apresentar a proposta e estágio atual de desenvolvimento de um museu virtual interativo sobre a história da computação, que pretende servir como apoio às aulas de introdução a computação em cursos de graduação da área, bem como de áreas afim. O museu, desenvolvido no ambiente virtual OpenSimulator, é composto por artefatos que perpassam por diferentes gerações da computação no que concerne a equipamentos, personalidades e linguagens de programação.

Entre as principais potencialidades e contribuições do museu apresentado, destacam-se a construção dos artefatos em três dimensões e as diferentes possibilidades de interação com os mesmos por parte dos estudantes, bem como integração com diferentes mídias. Desta forma, o Museu da Computação constitui-se em um espaço para a compreensão dos conteúdos introdutórios da computação e tem como inovação a possibilidade não somente de visualizar os objetos e conceitos de um computador, mas também de interagir com interfaces 3D e ambientes imersivos. Outro diferencial é a possibilidade de integração do museu a um ambiente virtual de 
ensino e aprendizagem, neste caso, o AVEA Moodle. Esta integração permite ao professor organizar todo seu conteúdo didático no Moodle e, através do mesmo, interagir com o museu virtual inserindo, a partir do AVEA.

No estágio atual, as demais salas estão sendo desenvolvidas, sendo que pretende-se avaliar o ambiente junto à turmas da disciplina de introdução ainda no segundo semestre de 2013 . A partir das avaliações realizadas, será possível analisar as reais contribuições do mesmo, bem como avaliar as possibilidades de melhorias. Ainda, encontra-se em fase de projeto e desenvolvimento avatares inteligentes que atuarão como guias de visitação ao museu, tal como em museus físicos. Esse recurso de agentes tem como objetivo potencializar as interações e aprendizagem colaborativa entre os estudantes dentro do museu.

\section{Referências Bibliográficas}

Almeida, J. M. F. (2006) "Museu Virtual de Informática". Universidade do Minho, Escola de Engenharia, Departamento de Sistemas de Informação.

Azevedo, C. E. F.; Elia, M. F. (2011) "Proposta de uma Aplicação de Mundos Virtuais na Educação usando o OpenSimulator com diferentes requisitos tecnológicos". Anais do XXII SBIE - XVII WIE.

Bainbridge, W. S. (editor). (2010) Online Worlds: Convergence of the Real and the Virtual. London: Springer.

Barris, K. (2007). To be virtual ...or not to be: Theatre museum Canada's travels into the world of technology. Canadian Theatre Review, (131), 49-52.

Berry, G; Sheard, J; Quartly, M. (2011). “A virtual museum of computing history: an educational resource bringing the relationship between people and computers to life". In: Proceedings of ACE '11. Australian Computer Society, Inc., Darlinghurst, Australia, Australia, 79-86.

Chengwei, Y., Chengle, Y., Shijun, L., Xiangxu, M. e Rui, W. (2011) "An Approach of Personalized 3D Scene Customization Based on Multimedia Resources" In International Conference on Multimedia and Signal Processing (CMSP), p. 131-135.

Fedelil, R. M., Giulio, E., Pollonil, F. PERES, F. (2003) Introdução à Ciência da Computação. São Paulo: Cengage Learning.

Forouzan, B., Mosharraf, F. (2012) Fundamentos da Ciência da Computação - Tradução da $2^{\mathrm{a}}$ Edição Internacional. São Paulo: Cengage Learning.

Giangrandi, P. and Mirolo, C. (2006) "Numeri e Macchine" - A virtual museum to learn thehistory of computing. In proceedings of the ITiCSE 2006, Bologna, Italy.

Lévy, P. Cibercultura. Lisboa : Instituto Piaget, 2000. p. 281.

Medina, E. M. (2004) "Beyond the ballot box: Computer science education and social responsibility". ACM SIGCSE Bulletin inroads 36(4), 7-10.

Osório, F. S.; Musse, S. R.; Santos, C. T.; Heinen, F.; Braun, A. e Silva, A.T. S. (2004). "Ambientes Virtuais Interativos e Inteligentes: Fundamentos, Implementação e Aplicações Práticas”. XXIV Congresso da SBC - JAI 2004. Tutorial. Salvador, Bahia.

Schlemmer, E.; Trein, D.; Oliveira, C. (2008) "Metaverso: a telepresença em Mundos Digitais Virtuais 3D por meio do uso de avatares". In: XIX Simpósio Brasileiro de Informática na Educação - SBIE 2008. Fortaleza - CE.

Wankel, C.; Kingsley, J. (2009) Higher Education in Virtual Worlds. Bingley: Emerald. 